\title{
Evaluación sobre el desempeño de docentes de un programa de Maestría
}

\author{
Assessment on the performance \\ of the Master's program's teachers
}

Informe

\author{
Celia Gabriela Villalpando Sifuentes \\ Universidad Autónoma de Ciudad Juárez - México \\ celia.villalpando@uacj.mx \\ Luis Arnulfo Ponce Loya \\ Centro Chihuahuense de Estudios de Posgrado - México \\ mtro_ponce@yahoo.com
}

Recibido: 28 de mayo de 2015 / Aceptado: 29 de enero de 2016

\section{Resumen}

El presente documento muestra el informe de una evaluación del desempeño docente realizada en México en un centro educativo de posgrado. El propósito de ésta es generar procesos de reflexión y mejora continua, a partir de la toma de decisiones que se puede desarrollar mediante el análisis y discusión de los resultados obtenidos. A través del mismo, se encuentran los argumentos que justifican la necesidad de éste, así como su finalidad. De igual manera, se logran identificar los referentes de la evaluación, en este caso atienden, como ya se mencionó, al desempeño de docentes de posgrado, resaltando cinco criterios para su valoración: Planeación, clima áulico, dominio disciplinar, dominio pedagógico y mecanismos de evaluación. El proceso evaluativo llevado a cabo se posiciona en el paradigma interpretativo, pues busca llegar a comprender e interpretar este fenómeno -el desempeño de los docentes de dicho programa educativo-; además, está orientada por el modelo iluminativo de Parlett y Hamilton. Dentro de las conclusiones se rescata, que la experiencia académica que se edifica en la institución, depende de la calidad y compromiso de los docentes que en él laboran.

Palabras clave: Evaluación de profesores, educación superior.

\section{Abstract}

The present document shows the report of an evaluation of the educational performance realized in an educational center of master degree. The intention of this one is to generate processes of reflection and improvement continues, from the capture of decisions that can develop by means of the analysis and discussion of the obtained results. Across the same one, they find the arguments that justify the need it, as well as his purpose. Of equal way, they are achieved to identify the modals of the evaluation, in this case they attend, since already it was mentioned, to the teachers' performance of master degree, highlighting five criteria for his valuation: To plan, climate's classroom, domain to discipline, pedagogic domain and mechanisms of evaluation. The process of evaluation carried out is positioned in the interpretive paradigm, since it seeks to manage to understand and interpret this phenomenon -the performance of the teachers of the above mentioned educational program-; besides is orientated by the illustrating model of Parlett and Hamilton. Inside the conclusions there is rescued, that the academic experience that is built in the institution, depends on the quality and commitment of the teachers working in it.

Keywords: Average rating of teachers, higher education.

Forma sugerida de citar: Villalpando, Celia y Ponce, Luis (2016). Informe de evaluación sobre el desempeño de docentes de un programa de Maestría. Alteridad, 11(1), pp. 78-87. 


\section{Introducción}

Resulta interesante partir de la afirmación que Santos (1998) expone:

La evaluación de los Centros escolares no suelen someterse a una evaluación sistemática y rigurosa. Las escuelas, sencillamente, funcionan. Cada año, cada día, se repiten las mismas rutinas sin saber muy bien por qué y para qué. La evaluación se realiza en ellas (no sobre ellas) para comprobar si los alumnos han aprendido lo que se les ha pretendido enseñar (p. 5).

Al hablar de evaluación, pareciera que el aprendizaje fuera el único ámbito susceptible de ser evaluado; sin embargo, también se puede evaluar sobre ella, sobre su clima escolar, la infraestructura, el currículum, por mencionar algunos ejemplos; y no solo se puede, sino que se debe hacer, pues este proceso valorativo debe enfrentar los retos exigidos por la sociedad actual relacionados con la calidad de la educación que se demanda a los planteles escolares. A través de este documento, en este caso, se expone la evaluación realizada al desempeño docente de un centro educativo de educación superior.

Para toda institución educativa resulta trascendental contar con información permanente y pertinente sobre sus procesos y resultados, lo cual da pie a efectuar modificaciones en el desempeño de todos aquellos que forman parte de la vida académica, a fin de reorientar adecuadamente sus acciones. Una institución educativa a nivel de posgrado que proporciona información de manera habitual tiene efectos positivos con respeto a su calidad, pues aporta datos que permiten saber, por un lado, en qué medida la actuación de los agentes educativos se desarrollan en grado satisfactorio y por otro, identificar cuáles acciones son necesarias reforzarse, con la finalidad de focalizar la atención en estas últimas, a miras de mejorar.

Partiendo de la idea de Castillo (2002), "la referencia a la calidad es, por tanto, también una referencia a la evaluación" (p. 115), por lo que se reconoce como crucial el rol de esta última dentro del marco de la educación, pues accede a valorar una realidad educativa desde todas sus aristas; a través de dicho acto busca abrir un abanico en el conocimiento de todo aquello que tenga que ver con la educación, con el compromiso de optimizar los recursos, el tiempo, los esfuerzos y la mejora permanente de estos. Cabe señalar, la evaluación es una tarea compleja, requiere conocimiento, comprensión a nivel holístico de los problemas y necesidades que toda actividad y hecho educativo exigen.

El propósito de la presente evaluación está encaminada a reconocer la medida en que se están logrando las metas planteadas para alcanzar la calidad educativa, por lo tanto, la información resultante del proceso evaluativo permitirá analizar la manera cómo funciona la institución, sus procesos internos, la forma de organización que le da vida a la participación y funciones de quienes laboran dentro de la misma. A nivel institucional, se busca a través de la evaluación, reflexionar sobre la práctica educativa propia y la de los otros, se pretende evaluar a fin de identificar no sólo áreas de oportunidad, sino también fortalezas en torno a aspectos específicos de la docencia, reconocer y organizar necesidades de formación tanto personales como del colectivo, esto con el compromiso de emprender un proceso de mejora continua en el desempeño docente.

De acuerdo al objeto de estudio a evaluar, se opta por elegir la evaluación focalizada al desempeño docente, ésta estudia y analiza la eficiencia del docente; su objetivo es encontrar indicadores que permitan a directivos, investigadores y a los mismos educadores la mejora de la calidad de la enseñanza, según Castillo (2003) permite la "valoración, análisis y toma de decisiones de mejora del conjunto de actuaciones educativas que lleva a cabo el profesorado en todos los ámbitos del sistema escolar para propiciar el aprendizaje formativo del alumnado y el desarrollo profesional del docente" (p. 82), idea que se busca por medio de este documento, para el centro educativo.

De acuerdo al uso que se le pretenden dar a los resultados del análisis de la información, esta evaluación tiene como finalidad orientarse a la 
toma de decisiones, pues pretende "proporcionar información a personas clave para [realizarlo]" (Valenzuela, 2004, p. 19), en este caso, la información generada de dicho proceso valorativo estará destinada al uso que de manera pertinente se haga en la dirección del centro educativo, ésta servirá de insumo para las decisiones requeridas de acuerdo a las necesidades de la institución, lo cual es fundamental para dicha acción con respecto a establecer metas, definir criterios, determinar acciones, todo encaminado hacia el mejoramiento de la institución.

De acuerdo a lo expresado, se concibe a la evaluación como un proceso que de forma sistemática obtiene información útil para emitir un juicio de valor, de acuerdo al fin perseguido. Puntualmente, para este proceso valorativo se pretende evaluar el cumplimiento de las funciones y responsabilidades áulicas de los docentes que laboran en el programa de maestría en cuestión. El destino de este informe tiene un principio formativo, ya que da la oportunidad al docente de situarse como miembro de la comunidad escolar, reflejarse en su práctica a través de las opiniones y juicios de quienes son sus colegas y estudiantes; lo cual servirá de plataforma para reflexionar y actuar en pro de una mejor práctica.

\section{Fundamentación teórica y metodológica}

\section{La evaluación como elemento clave para conocer el desempeño docente}

El acto de evaluar una institución da paso a la indagación del docente, del alumno, de sus interacciones, del currículum y del contexto; todo esto con la finalidad de descubrir cómo influyen y constituyen el hecho educativo. El quehacer del docente se circunscribe dentro de las aulas, no obstante, su labor no se puede aislar, contrario a ello, es requerimiento compartir y socializar tanto sus experiencias como sus preocupaciones, de ahí la necesidad de hacer uso del presente informe para ser partícipe de ese entramado de reflexiones que den pie a la toma de decisiones encaminadas a mejorar los procesos educativos de la institución. En este caso, una de las direcciones de este esfuerzo consiste en evaluar el desempeño docente, evidentemente a través de la búsqueda en su quehacer educativo. Debido a lo complicado del tema, se hace necesario presentar algunas posturas y concepciones en torno a éste, vale señalar que existen concepciones que van desde la idea de la transmisión de conocimientos hasta el despertar de la consciencia reflexiva, pero en esta ocasión, el sustento teórico no se centrará en la trasmisión.

Para Bazdresch (2000), la práctica docente es una práctica intencionada, implica todo eso que el docente pone en juego para facilitar que el estudiante se apropie de los contenidos en un proceso creativo. Por su parte, Guzmán (2005) plantea tres factores, los cuales en correlación hacen posible impulsar una buena docencia, primero se presenta la necesidad de tener dominio de los contenidos, le sigue la motivación que el docente despierta y le acompaña la habilidad comunicativa. Contextualizando esto, un profesor de posgrado requiere además de cierta habilidad para enseñar un conocimiento disciplinario, estimular al alumno a desarrollar el pensamiento, a fin de lograr la independencia intelectual, esto para considerarse un buen docente. En este mismo orden de ideas, Fierro, Fortoul y Rosas (1999) afirman:

La práctica docente trasciende la concepción técnica de quien sólo se ocupa de aplicar técnicas de enseñanza en el salón de clases. El trabajo del maestro está situado en el punto en que se encuentran el sistema escolar... y los grupos sociales particulares, en este sentido su función es mediar el encuentro entre el proyecto político educativo, estructurado como oferta educativa, y sus destinatarios, en una labor que se realiza cara a cara (pp. 20 -21).

Dentro de esta concepción se ve a la práctica docente como una praxis social, en la cual juegan un papel fundamental los significados, las percepciones y las acciones de los docentes, estudiantes, autoridades educativas y del proyecto educativo que concreta la función del maestro. Hace más de cuatro 
décadas Kosik (como se citó en Perales, 2006) ya rescataba la idea de acción docente como actividad transformadora, al expresar "la práctica -en el sentido de la praxis- se constituye de acciones interpretadas que para ser consideradas educativas, necesariamente deben ser acciones transformadoras" (p. 25), en la actualidad dicha idea se rescata al momento de considerar la práctica como el conjunto de acciones que intencionadamente propician la transformación de los sujetos partícipes en ella. Para la valoración de este ámbito, se habrán de considerar los criterios de planeación, clima áulico, dominio disciplinar, dominio pedagógico y mecanismos de evaluación.

En el último decenio, los sistemas educativos latinoamericanos han identificado el desempeño profesional del maestro como un factor determinante del logro de la calidad de la educación. Diversos estudios concluyen que podrán perfeccionarse los planes de estudio, programas de textos escolares, construirse magníficas instalaciones; obtener excelentes medios de enseñanza, pero sin docentes eficientes, no podrá tener lugar el perfeccionamiento de la educación (Ministerio de Educación Nacional, 2003, p. 7).

De ahí la necesidad de evaluar el desempeño docente a partir de los siguientes criterios:

\section{Criterios para evaluar el desempeño docente}

Con respecto a la planeación, según la SEP (2013a) "se trata de asegurar que cada profesor desarrolle con claridad su programa de estudio... deberá establecer cómo se percata de los avances de los alumnos y de las dificultades que se deben superar... debe ser pertinente y viable" (p. 13). Su importancia radica en la manera de orientar los procesos para el desarrollo de la enseñanza y el aprendizaje hacia la calidad educativa, por tal razón, se quiere conocer si el docente prepara sus clases con antelación.

En relación al criterio del clima áulico; entendiéndose éste "como el conjunto de actitudes generales hacia y desde el aula, de tareas formativas que se llevan a cabo por el profesor y los alumnos y que definen un modelo de relación humana en la misma" (Quispe, Pérez y Pérez, s. f., p. 1), en este caso, se considerarán principalmente las conductas y las actitudes que fomenta y desarrolla el docente dentro de la clase, teniendo en cuenta que éste "queda condicionado por el tipo de prácticas que se realizan en el aula... por la personalidad e iniciativa del profesor, por la homogeneidad o heterogeneidad del grupo, y por el espíritu subyacente en toda la institución" (Quispe, Pérez y Pérez, s. f., p. 1).

Específicamente, para el criterio del dominio disciplinar, es precisamente, donde se ubica el conocimiento del docente sobre la o las asignaturas que imparte.

Diferentes autores incluyen esta competencia entre las necesarias para ser maestro. Por ejemplo, Cano García... al referirse a las diferentes clasificaciones de competencias docentes, en todas ellas se señala, con distintas denominaciones, el dominio de los contenidos: - Competencia académica (dominio de los contenidos de su área) y competencia didáctica (manejo de los componentes del proceso de enseñanza-aprendizaje) - Adquisición de conocimientos sobre la disciplina y su didáctica - Competencia cultural (conocimiento de la materia y la cultura, en general) y competencia pedagógica (habilidades didácticas: dinamizar grupos,...) (López, 2007, p. 42).

En este criterio se conocerá la claridad del docente con respecto a la finalidad educativa.

Bajo el nombre de dominio pedagógico se encuentra una serie de indicadores que buscan conocer la organización de la clase que hace el docente con respecto al trabajo en el aula, con base en lo indicado por Díaz-Barriga y Hernández (2001) "el uso de estrategias de enseñanza lleva a considerar al agente de enseñanza, especialmente en el caso del docente, como un ente reflexivo, estratégico... que puede ser capaz de proponer... una enseñanza estratégica" (p. 2). De ahí la importancia de recuperar el empleo que se hace de diferentes estrategias y herramientas didácticas para favorecer los procesos de aprendizaje de sus estudiantes. 
Por último, se evaluará el criterio de mecanismos de evaluación; considerando que:

La evaluación es un proceso integral y sistemático a través del cual se recopila información de manera metódica y rigurosa, para conocer, analizar y juzgar el valor de un objeto educativo determinado: los aprendizajes de los alumnos, el desempeño de los docentes... (SEP, 2013b, p. 19).

Dentro de este se busca rescatar la importancia dada a la evaluación dentro de los procesos de enseñanza-aprendizaje.

\section{Estrategia metodológica}

Todo lo que tenga que ver con educación, procesos educativos, calidad educativa, docentes, alumnos, directivo, gestión escolar, programas o consejo técnico, puede ser potencialmente evaluado de una u otra forma. Cualquier tipo de evaluación desarrollada dentro de las instituciones educativas requiere de un proceso indagatorio específico, su fin es el de conocer la realidad de cualesquier acto inmerso en la educación. En la actualidad, la naturaleza de la evaluación educativa se caracteriza por su complejidad, lo cual la hace difícil de entender en toda su magnitud, obedeciendo a lo expuesto, para este caso, se presentará una estrategia metodológica que guiará el presente proceso evaluativo.

Se pretende posicionar para el desarrollo de la presente evaluación en el paradigma interpretativo, cuyas características se gestan en las diversas perspectivas subjetivas. Desde esta filosofía se reconoce la necesidad de recuperar las percepciones, opiniones e ideologías que dan vida a los significados e interpretaciones que los individuos construyen de sus interacciones.

El propósito de la evaluación cualitativa es comprender la situación objeto de estudio mediante la consideración de las interpretaciones, intereses y aspiraciones de quienes en ella interactúan, para ofrecer la información que cada uno de los participantes necesita en orden de entender, interpretar e intervenir del modo más adecuado ("Paradigmas y modelos de la evaluación educativa”, s. f., p. 9).

Lo que se busca con este tipo de paradigma, es llegar a la comprensión de fenómenos educativos, en este caso, lo que envuelve al desempeño docente; intentando interpretar la información ofrecida por los docentes, directivos y estudiantes, de acuerdo a sus experiencias y vivencias con respecto al centro educativo donde se desarrolla la dinámica en cuestión. Otra de las bondades brindadas desde esta postura es la posibilidad que ofrece a los agentes educativos de analizar y reflexionar sobre sus propios procesos, con la finalidad de realizar las modificaciones idóneas, a fin de lograr alcanzar los objetivos planteados.

En este caso particular se guiará con el modelo de evaluación iluminativa de Parlett y Hamilton (1977), las características que hicieron posible su elección tienen que ver con la comprensión holística requerida para el objeto a evaluar, reconoce el contexto donde se desarrolla de manera amplia y la evaluación la efectúa en condiciones naturales o como se le conoce, de campo, además, apuesta a la interpretación del desarrollo evaluativo y del análisis de los procesos, asimismo alienta a recolectar información por medio de entrevistas, cuestionarios, observación y análisis de documentos. Cabe señalar:

La evaluación iluminativa no es un método regularizado sino una estrategia global que puede adoptar diversas formas. Pretende ser adaptable para descubrir las dimensiones, metas y técnicas de evaluación, y ecléctica en el sentido de proporcionar al evaluador una serie de técnicas investigativas, que dependerán del problema que deba investigarse (Jiménez et al., 1999, p. 51).

La presente evaluación se desarrolló en una institución educativa de educación superior, específicamente en un programa de maestría relacionada con la educación. Los sujetos participantes en este proceso valorativo representa la totalidad de su población, esto es, 79 estudiantes que cursan primer, segundo, tercer y cuarto semestre; asimismo dos directivos y 12 docentes que laboran en el plantel. Las técnicas empleadas para la recogida de 


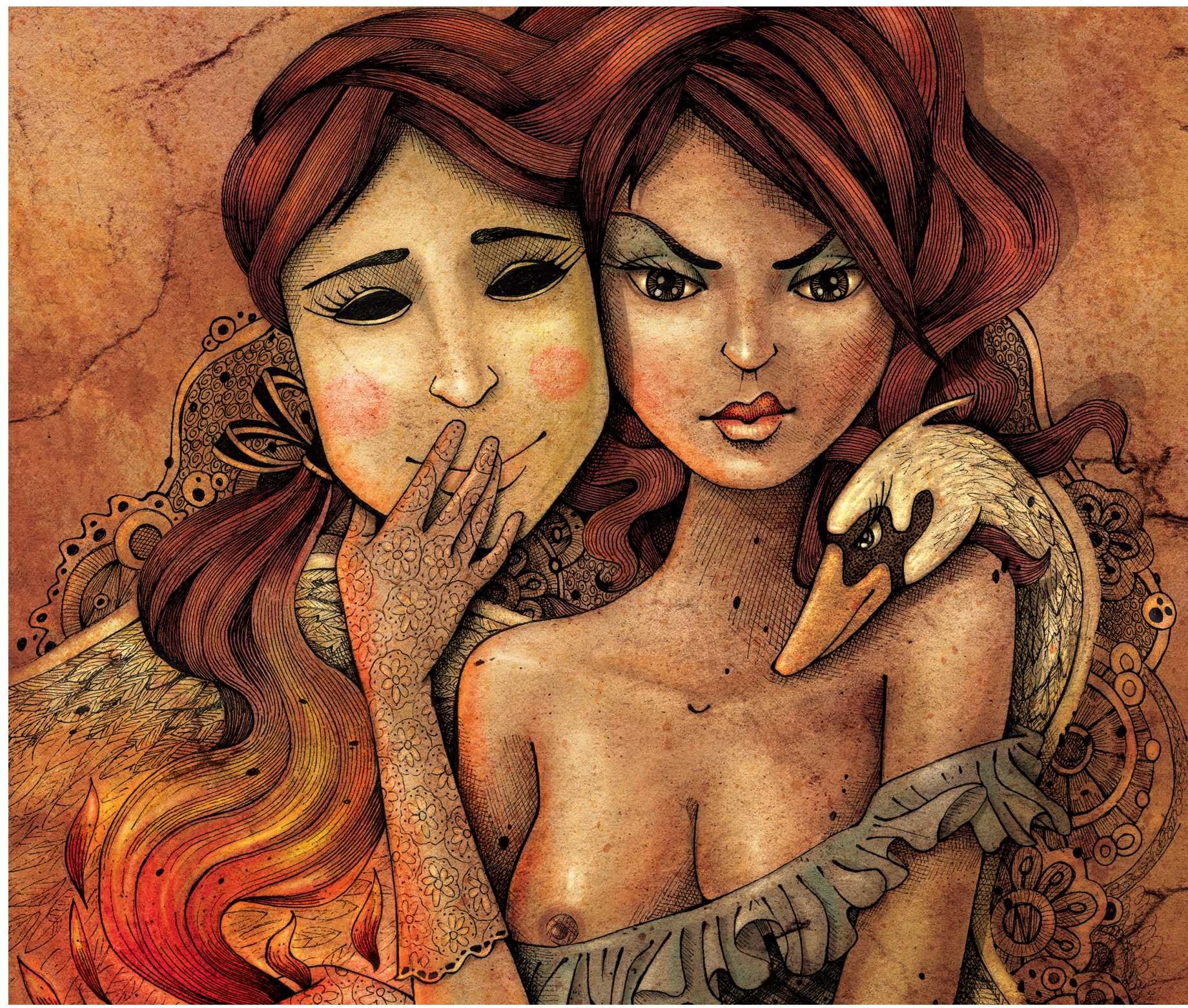

OTRO YO POR MI MISMO Mixta/ plumilla sobre papel y

retoque digital

datos fueron entrevistas a directivos y docentes, observaciones a las sesiones de trabajo y cuestionarios para los estudiantes. A fin de hacer el análisis de la información se realizó una triangulación de datos con el fin de verificar y comparar la información obtenida; en palabras de Okuda y Gómez-Restrepo (2005) "es una herramienta enriquecedora que le confiere a un estudio rigor, profundidad, complejidad y permite dar grados variables de consistencia a los hallazgos" (p. 123).

\section{Análisis y resultados}

Resulta interesante enfatizar que algunos de los maestrantes, a fin de valorar el desempeño de sus docentes, echan mano de ciertas características personales: 
Es una maestra simpatiquísima (Estudiante7 Primer semestre).

Su vestuario me parece inapropiado (Estudiante 19 Tercer semestre).

Contrario a lo que Bazdresch (2000), expone en relación a que la práctica docente es una práctica intencionada, implica todo eso que el docente pone en juego para facilitar que el estudiante se apropie de los contenidos en un proceso creativo. No obstante, también rescatan elementos relacionados con el quehacer docente; en relación al criterio de la planeación, y con base en lo expuesto por la SEP (2013a) "se trata de asegurar que cada profesor desarrolle con claridad su programa de estudio" (p. 13); en este caso se hace evidente en la mayoría de los docentes el uso de este instrumento para el desarrollo de la enseñanza y el aprendizaje en sus aulas. Sus prácticas son congruentes con lo estipulado en el Programa de estudios de la maestría, y la organización de los tiempos posibilita el cumplimiento de los contenidos programados con antelación; empero, ninguno de ellos realiza adecuaciones curriculares requeridas para la atención de algún estudiante en particular.

Con respecto al clima áulico, éste "queda condicionado por el tipo de prácticas que se realizan en el aula... por la personalidad e iniciativa del profesor, por la homogeneidad o heterogeneidad del grupo" (Quispe, Pérez y Pérez, s. f., p. 1). En la mayoría de los casos tanto los estudiantes como los docentes manifiestan sentirse cómodos con el tipo de relaciones que se establecen en las aulas, vivencian un ambiente de confianza, por lo tanto trabajan de manera cordial y respetuosa. Pese a eso, en uno de los casos, casi el 100\% de los maestrantes puso al descubierto una situación alarmante en relación al trato recibido de uno de sus asesores expresando que éste "genera un clima tenso y de miedo», temen expresar sus opiniones debido a su reacción. Hacen patente un panorama carente de interacción, tolerancia y diálogo, plagado de autoritarismo y faltas de respeto, lo que genera un espacio intimidante y obstaculizador para el desarrollo académico.
A fin de comprender ese complejo entramado de relaciones que se dan en el aula, el docente debe acercarse al cómo sucede y por qué suceden las diversas situaciones que comprenden el llamado clima áulico. Dicha complejidad solo puede reconocerse desde el origen multifactorial de los problemas.

Hay varios aspectos que determinan el clima del aula, entre ellos el ambiente de aprendizaje, los procesos de comunicación y la disciplina. El orden interior, fruto de una buena organización del trabajo, favorece así mismo el desarrollo de la disciplina interna y el crecimiento y la vivencia de experiencias educativas cargadas de significados (Cora, 2007, p. 15).

Idea que se ve reflejada en los salones durante las diferentes clases impartidas en la maestría, en dicha institución educativa.

A pesar de que el alumnado piensa que sus docentes tienen buen dominio de los contenidos de las asignaturas, consideran que en gran parte de los casos, les falta atender dentro de sus procesos de enseñanza la manera como explican algunos conceptos o ideas, a fin de dinamizar la construcción de significados, situación que requiere una atención especial, pues en palabras de López (2007) dicha competencia es fundamental para ser maestro, empero, tanto los docentes, los directivos y los estudiantes están conformes con la organización de las clases: "Generalmente elijo las asignaturas que me gustan, con las que me siento cómodo y que además conozco los contenidos" (Docente 5).

Díaz-Barriga y Hernández (2001) manifiestan que "el uso de estrategias de enseñanza lleva a considerar al... docente, como un ente reflexivo, estratégico... que puede ser capaz de proponer... una enseñanza estratégica" (p. 2). Con respecto al dominio pedagógico, su rol se reduce a tomar las decisiones del rumbo que habrán de llevar sus clases, pues seleccionan actividades donde pide a los alumnos la explicación, argumentación o cualquier tipo de aportación con respecto al tema, por su parte el profesor recoge éstas para ampliar, 
explicar o clarificar las ideas. El contrato didáctico y la negociación entre docentes y alumnos durante la implementación curricular se manifiesta con acciones que reconocen la responsabilidad que tienen el docente frente al estudiante y viceversa. La organización del grupo se da de acuerdo al contenido; y las modalidades de trabajo son: individual, binas, equipos o grupal. En cuanto a los mecanismos de evaluación desarrollados en el aula, los estudiantes consideran que la parte del diálogo con respecto a estos procesos, aún se encuentra débil, en el sentido de no recibir retroalimentación sobre su desempeño. Empero, no se logra identificar esa "enseñanza estratégica» de la cual hablan los autores.

Kosik (como se citó en Perales, 2006) ya rescataba la idea de acción docente como actividad transformadora, al expresar "la práctica -en el sentido de la praxis- se constituye de acciones interpretadas que para ser consideradas educativas, necesariamente deben ser acciones transformadoras" (p. 25), en el caso de la maestría en cuestión se identifican más prácticas tradicionalistas que transformadoras.

\section{Discusión y conclusiones}

"Interesarse por investigar la vida cotidiana en la escuela es un reto que implica adentrarse a un mundo de interacciones, simbolismos, signos, rituales y procesos de construcción de significados" (Quiroz, 2009, p. 16). En el caso de esta evaluación, a nivel metodológico se considera como fortaleza la flexibilidad y bondades que ofrecen tanto el paradigma interpretativo como el modelo iluminativo, pues permitieron establecer una relación estrecha entre los evaluadores y los participantes, además dieron oportunidad de reconocer la realidad como múltiple, permitiendo de esta manera su estudio holístico, por otro lado, se tuvo la posibilidad de emplear métodos de recolección de información no estandarizado lo cual facilitó el proceso.

El docente asume su rol con un alto grado de responsabilidad con respecto al cumplimiento que debe tenerse en la práctica educativa, pues considera que lo indicado en el programa de la (s) asignatura (s) impartidas, es lo que los estudiantes de posgrado habrán de aprender en este nivel educativo. Los docentes reconocen que deben desarrollar habilidades, capacidades y actitudes en sus estudiantes a partir de lo señalado en estos, afirman realizar una planeación acorde a las necesidades de la materia, mas no a las características e individualidades de sus estudiantes, de lo cual estos últimos concuerdan con sus asesores.

No obstante, en su mayoría los estudiantes refieren una buena práctica en la docencia, considerando actuaciones dignas de un asesor de posgrado. Valoran el dominio de la materia, independientemente de la personalidad del docente, así mismo rescatan como elemento toral para una dinámica áulica de calidad, la interacción establecida durante el manejo de los contenidos de la asignatura; sobre todo el ambiente de respeto en el espacio de trabajo, así como la accesibilidad para atender dudas y la transparencia en los mecanismos de evaluación.

Numéricamente hablando, el estudiantado del centro escolar valora el desempeño de sus docentes con un 4.6/5; mientras que los propios docentes se promedian con un 4.8/5. Coinciden, tanto docentes como estudiantes, en que la mayor fortaleza en la institución son las relaciones cordiales y el respeto generado en el clima áulico. Los indicadores que resultaron más bajos en la evaluación, desde la visión del estudiante, en primer lugar, es que no se considera su opinión para mejorar la clase, el docente no enfatiza los aspectos positivos de su desempeño, y como tercer punto, no se le informa periódicamente sobre su evaluación. Descriptivamente, ese 4.6 se podría traducir, a través de la mirada de los maestrantes, en expresiones como la siguiente:

Mi más profundo agradecimiento y el más grande reconocimiento al trabajo que ha venido realizando la maestra, desde mi muy humilde opinión una de las fortalezas, más representativas de la institución (Estudiante 20 Cuarto semestre).

La experiencia académica que se edifica en el centro educativo, depende de la calidad y compro- 
miso de los docentes que en él laboran. De acuerdo a los directivos del centro, ellos son "profesionales, solidarios". Sin embargo, cuando se alude a la calidad de una institución, los docentes habrán de:

Mantenerse actualizados a fin de llevar la delantera en el conocimiento -su construcción y aplicación- sobre la realidad educativa. La actualización refiere al conocimiento de las materias, dinámica de trabajo, estrategias didácticas (Directivo 1).

Tal y como lo mencionan Fierro, Fortoul y Rosas (1999), al afirmar que la práctica trasciende la concepción técnica de la enseñanza; por lo tanto, es fundamental crear alternativas de mejoramiento encaminadas a comprometer a los docentes a transformar su quehacer, de ahí que la evaluación se relacione con la acción y con la reflexión, ejercicio que posibilita ahondar en la comprensión de la práctica educativa; a fin de alejarse de ese actuar técnico que reduce la evaluación solo a la obtención de resultados. La intención se acerca a la:

Estrategia 'evaluar para mejorar' [la cual] busca construir una cultura que permita ver la evaluación como una herramienta que lleva a la reflexión sobre la acción realizada y los resultados obtenidos, con el fin de diseñar planes de mejoramiento institucional encaminados a superar de manera sistemática las dificultades en el alcance de los logros (Ministerio de Educación Nacional, 2003, p. 5).

$\mathrm{Al}$ retomar el propósito de la presente evaluación, este documento posibilitó a los miembros de la institución la oportunidad de reflexionar sobre el desempeño docente que ahí se gesta, con el fin de reconocer tanto sus fortalezas como sus debilidades, mismas que permitieron identificar que la docencia en este programa de maestría, actualmente goza de más aspectos positivos que negativos. La información obtenida fue facilitada a la dirección del centro educativo, a fin de diseñar alguna estrategia pertinente que permita tomar las decisiones correctas para el mejoramiento académico.

\section{Referencias bibliográficas}

Bazdrech, M. (2000). Vivir la educación, transformar la práctica. México: Textos educar-Educación Jalisco.

Castillo, S. (Coord.). (2002). Compromisos de la evaluación educativa. Madrid: Pearson Educación, S. A. (2003). Vocabulario de Evaluación Educativa. Madrid: Pearson Educación, S. A.

Cora, M. R. (2007). El clima del aula: un abordaje desde la ética. Cuadernos de Investigación Educativa, 2(14), 7-27. Universidad ORT Uruguay.

Díaz-Barriga, F. y Hernández, R. (2001). Estrategias docentes para un aprendizaje significativo. México: Mc Graw Hill.

Fierro, C., Fortoul, B. y Rosas, L. (1999) Transformando la práctica docente. Una propuesta basada en la investigación-acción. México: Maestros y Enseñanza Editorial Paidós Mexicana, S.A.

Guzmán, C. J. (2005). El profesor efectivo en educación superior. En: F. Fierro y M. H. García (Comps.), Pensamiento didáctico y práctica docente (pp.15-67). México: UNAM.

Jiménez, B., et al. (1999). Evaluación de programas, centros y profesores. Madrid. Síntesis Educación. Bogotá.

López, A. (Coord.). (2007). El desarrollo de competencias docentes en la formación del profesorado. Madrid: MEC.

Ministerio de Educación Nacional (2003). Manual de la evaluación de desempeño.

Okuda, B. M. y Gómez-Restrepo, C. (2005). Métodos de investigación cualitativa: triangulación. Revista Colombiana de Psiquiatría, XXXIV(1), 118124. Asociación Colombiana de Psiquiatría. Bogotá, D. C., Colombia.

Paradigmas y modelos de evaluación educativa. (s.f.). Recuperado de: http://roble.pntic.mec. es/ agarci19/asignatura/temauno.htm

Perales, P. R. (Coord.) (2006). La significación de la práctica docente. México: Editorial Paidós Mexicana, S.A.

Quiroz, M. S. (2009). Epistemologías de la vida cotidiana escolar. Mitos, rituales, creencias, hábitus, imaginarios. México.

Quispe, G. L., Pérez, O. N. y Pérez, I. C. (s.f.). Clima escolar áulico: un estudio desde las tutorías. Universidad Nacional de Jujuy, Facultad de Ingeniería. 
Santos, M. Á. (1998). Hacer visible lo cotidiano. Teoría y práctica de la evaluación cualitativa de los centros escolares. 3a. Ed. España: Ediciones Akal, S. A.

Secretaría de Educación Pública (SEP) (2013a). Lineamientos para la organización y el funcionamiento de los consejos técnicos escola- res. Educación Básica. Preescolar. Primaria. Secundaria. México.

(2013b). El enfoque formativo de la evaluación. México.

Valenzuela, R. (2004). Evaluación de instituciones educativas. México: Trillas 